\title{
A Neutralizing Antibody Assay Based on a Reporter of Antibody-Dependent Cell-Mediated Cytotoxicity
}

\author{
Yuling Wu, ${ }^{1,4}$ Jia J. Li, ${ }^{1}$ Hyun Jun Kim, ${ }^{2}$ Xu Liu, ${ }^{1}$ Weiyi Liu, ${ }^{1}$ Ahmad Akhgar, ${ }^{1}$ Michael A. Bowen, ${ }^{3}$ Susan Spitz, ${ }^{1}$ \\ Xu-Rong Jiang, ${ }^{2}$ Lorin K. Roskos, ${ }^{1}$ and Wendy I. White ${ }^{1}$
}

Received 27 March 2015; accepted 10 June 2015; published online 24 July 2015

\begin{abstract}
Benralizumab is a humanized anti-IL5 receptor $\alpha(\operatorname{IL} 5 \mathrm{R} \alpha)$ monoclonal antibody (mAb) with enhanced (afucosylation) antibody-dependent cell-mediated cytotoxicity (ADCC) function. An ADCC reporter cell-based neutralizing antibody (NAb) assay was developed and characterized to detect $\mathrm{NAb}$ against benralizumab in human serum to support the clinical development of benralizumab. The optimal ratio of target cells to effector cells was 3:1. Neither parental benralizumab (fucosylated) nor benralizumab Fab resulted in ADCC activity, confirming the requirement for ADCC activity in the $\mathrm{NAb}$ assay. The serum tolerance of the cells was determined to be $2.5 \%$. The cut point derived from normal and asthma serum samples was comparable. The effective range of benralizumab was determined, and $35 \mathrm{ng} / \mathrm{mL}$ [ $80 \%$ maximal effective concentration $\left(\mathrm{EC}_{80}\right)$ ] was chosen as the standard concentration to run in the assessment of NAb. An affinity purified goat anti-benralizumab polyclonal idiotype antibody preparation was shown to have NAb since it inhibited ADCC activity in a dosedependent fashion. The low endogenous concentrations of IL5 and soluble IL5 receptor (sIL5R) did not demonstrate to interfere with the assay. The estimated assay sensitivities at the cut point were 1.02 and $1.10 \mu \mathrm{g} / \mathrm{mL}$ as determined by the surrogate neutralizing goat polyclonal and mouse monoclonal anti-drug antibody (ADA) controls, respectively. The assay can detect NAb (at $2.5 \mu \mathrm{g} / \mathrm{mL}$ ) in the presence of $0.78 \mu \mathrm{g} / \mathrm{mL}$ benralizumab. The assay was not susceptible to non-specific matrix effects. This study provides an approach and feasibility of developing an ADCC cell-based NAb assay to support biopharmaceuticals with an ADCC function.
\end{abstract}

KEY WORDS: ADCC; benralizumab; cell-based assay; mechanism of action; neutralizing antibody.

\section{INTRODUCTION}

Immunogenicity assessments are an essential component of safety evaluation in the clinical development of biopharmaceuticals. Assessing anti-drug antibodies (ADAs) for neutralizing activity is a key characterization step since neutralizing ADAs may lead to altered pharmacokinetic (PK) and pharmacodynamic (PD) profiles $(1,2)$, reduced drug efficacy, and adverse events $(3,4)$.

Neutralizing antibody (NAb) assays are commonly classified into two types: ligand binding assay and cell-based assay. A ligand binding NAb assay typically assesses the binding of the drug to its target (e.g., soluble recombinant receptor). A cell-based NAb assay involves cells expressing the intended target of the drug. The NAb interferes with the

\footnotetext{
${ }^{1}$ Clinical Pharmacology and DMPK, MedImmune LLC, One MedImmune Way, Gaithersburg, Maryland 20878, USA.

${ }^{2}$ Analytical Biotechnology Development, MedImmune LLC, One MedImmune Way, Gaithersburg, Maryland 20878, USA.

${ }^{3}$ Antibody Development and Protein Engineering, MedImmune LLC, One MedImmune Way, Gaithersburg, Maryland 20878, USA.

${ }^{4}$ To whom correspondence should be addressed. (e-mail: wuyu@medimmune.com)
}

mechanism of action (MoA) of the drug, resulting in altered functional activities of cells and, hence, a modification of the assay readout signal. The cellular responses exhibited in cellbased assays may be more closely related to the ways in which NAbs are affected with the drug in vivo, and therefore, a cell-based NAb assay may be the preferred platform for evaluating NAb activity (5). However, disadvantages of a cellbased NAb assay have been generally recognized including labor intensive, low throughput, and less robustness compared to a ligand binding NAb assay. Laboratory assessments may include a comparison of NAb data generated in both ligand binding assay and cell-based assay formats.

Cell-based NAb assays have been developed to advance biopharmaceuticals through clinical development $(6,7)$. Onestep cell-based NAb assays allow both drug activity and drug NAbs to be quantified rapidly (6). Each NAb assay is designed and developed based on the unique MoA of each biopharmaceutical. Antibody-dependent cell-mediated cytotoxicity (ADCC) is a common MoA for therapeutic antibodies when they are designed to deplete cellular targets (8). The fragment antigen binding (Fab) domains of the antibody bind to their antigens on target cells, and the fragment crystallizable $(\mathrm{Fc})$ region of the antibody interacts with the FcyRIIIa (CD16) receptor on effector cells including natural killer 
(NK) and other myeloid cells, resulting in the release of cytokines (i.e., IFN $\gamma$ ) and cytotoxic granules (i.e., perforin and granzymes), leading to ADCC $(9,10)$.

Benralizumab is a humanized afucosylated monoclonal antibody $(\mathrm{mAb})$ against IL5 receptor $\alpha(\mathrm{IL} 5 \mathrm{R} \alpha)$ with enhanced effector function. It potently induces ADCC of eosinophils and basophils, selectively depleting circulating and tissue-resident eosinophils and basophils. To support the clinical development of benralizumab for the treatment of asthma and chronic obstructive pulmonary disease (COPD), we developed NAb assays in two platforms, ligand binding assay and cell-based assay formats. We have successfully validated both assays and subsequently conducted a side by side comparison using a subset population of clinical study samples (data not shown). Our data demonstrated advantages of ligand binding NAb assay in many aspects and supported that ligand binding NAb assay is the choice for the pivotal trials.

An ADCC reporter is employed in the cell-based NAb assay and is intended to reflect the MoA of benralizumab. This study presents the development, optimization, and characterization of an ADCC cell-based NAb assay. To our knowledge, our assay is the first cell-based NAb assay for the detection of NAb against a mAb with an ADCC MoA. Assay development work yielded comparable results to validation runs, confirming the robustness and suitability of the assay for downstream application.

\section{MATERIALS AND METHODS}

\section{Materials}

The CTLL-2/IL5R $\alpha$ cell line and NK92/NFAT-luciferase cell line were generated internally by MedImmune LLC. Benralizumab, benralizumab Fab, parental benralizumab (fucosylated) MEDI-563, and isotype controls were generated by MedImmune LLC. Goat anti-benralizumab polyclonal antibody was produced and purified (through a Protein A affinity column followed by a drug affinity column) by MedImmune LLC. Individual healthy, asthma serum samples and pooled normal human serum (NHS) were purchased from Bioreclamation. RPMI 1640 with GlutaMaxTM, horse serum (heat inactivated), human IL5, geneticin, and mercaptoethanol were purchased from Invitrogen. Fetal bovine serum (FBS, heat inactivated) was purchased from HyClone. Steady-Glo ${ }^{\circledR}$ Luciferase Assay System was purchased from Promega. Human IL-2 was purchased from Henry Schein. Human IL5 ELISA kit was purchased from R\&D Systems. Human soluble IL5R immunoassay was developed by the Bioanalytical Sciences group at MedImmune LLC.

\section{Cell Culture}

\section{CTLL-2/IL5R $\alpha$ Cells}

CTLL-2 is a murine cytotoxic $\mathrm{T}$ cell line, engineered to express human IL5R $\alpha$. CTLL-2/IL5R $\alpha$ cell growth medium contains $10 \%$ FBS, $0.5 \mathrm{mg} / \mathrm{mL}$ geneticin, $1 \mathrm{mM}$ sodium pyruvate, and $2 \mathrm{ng} / \mathrm{mL}$ human IL5 in RPMI 1640 GlutaMax. $1.0 \times 10^{4}$ cells $/ \mathrm{mL}$ were seeded and maintained for 3 days in approximately $50 \mathrm{~mL}$ growth medium in a T-175 cell culture flask or equivalent in a humidified incubator at $37 \pm 2^{\circ} \mathrm{C}$ with $5 \pm 1 \% \mathrm{CO}_{2}$. When the cell density was within the target range of $2.0 \times 10^{5}$ to $1.0 \times 10^{6}$ cells $/ \mathrm{mL}$, the cells were ready for passaging and testing in the assay.

\section{NK92/NFAT-Luciferase Cells}

NK92/NFAT-luciferase is a human cytotoxic natural killer cell line, engineered to express CD16 and NFATluciferase reporter gene constructs. The NK92/NFAT-luciferase cell growth medium contains $12.5 \%$ FBS, $12.5 \%$ horse serum, $2 \mathrm{mM}$ L-glutamine, $0.18 \%$ 2-mercaptoethanol, $0.5 \mathrm{mg} / \mathrm{mL}$ geneticin, and $100 \mathrm{IU} / \mathrm{mL}$ human IL-2 in RPMI 1640 GlutaMax. $4.0 \times 10^{4}$ cells $/ \mathrm{mL}$ were seeded and maintained for 3 days in approximately $50 \mathrm{~mL}$ growth medium in a T-175 cell culture flask or equivalent in a humidified incubator at $37 \pm 2^{\circ} \mathrm{C}$ with $5 \pm 1 \% \mathrm{CO}_{2}$. When the cell density was within the target range of $2.0 \times 10^{5}$ to $1.0 \times 10^{6}$ cells $/ \mathrm{mL}$, the cells were ready for passaging and testing in the assay.

\section{Methods}

\section{Assay Procedure}

Target cells are CTLL-2/IL5R $\alpha$ cells, a murine cytotoxic cell line stably transfected with IL5R $\alpha$. NK92/NFAT-luciferase cells (the effector cells of ADCC) are human NK cells that have been dually transfected with both CD16 and luciferase (the reporter gene that is under the control of a NFAT promoter).

Diluted serum samples were pre-incubated with benralizumab for $60 \pm 5 \mathrm{~min}$ at room temperature (RT) in a 96-well v-bottom polypropylene plate, and the mixture was transferred to a white polystyrene microplate. CTLL-2/ hIL5R $\alpha$ cells $\left(6 \times 10^{4} /\right.$ well $)$ and NK92/NFAT-luciferase cells $\left(2 \times 10^{4} /\right.$ well $)$ were washed, counted, and added to each well of the plate. The mixture of serum sample, benralizumab, and cells was incubated in a humidified cell culture incubator $\left(37 \pm 2^{\circ} \mathrm{C}\right.$ with $\left.5 \pm 1 \% \mathrm{CO}_{2}\right)$ for $4.5 \mathrm{~h}$. Steady-Glo ${ }^{\circledR}$ was added to each well and incubated for $60 \pm 5 \mathrm{~min}$ at RT.

The relative luminescence units (RLU) per well were measured with a luminometer (PerkinElmer EnVision ${ }^{\mathrm{TM}}$ Multilabel Reader). The RLU is proportional to the ADCC activity and inversely proportional to the levels of neutralizing ADA present in serum samples. Responses of samples were compared to a cutoff RLU value. Samples with an RLU response above the cutoff were declared negative for neutralizing ADA. Samples with RLU responses equal to or below the cutoff were positive for NAb.

\section{Quality Control Sample Preparation}

The ADCC NAb assay included cell control that only comprised CTLL-2/IL5R $\alpha$ cells and NK92/NFAT-luciferase cells in the assay medium to represent the background in the assay medium. The serum control comprised CTLL-2/IL5R $\alpha$ cells and NK92/NFAT-luciferase cells in 2.5\% NHS to represent the background in the assay matrix. Negative quality control (NQC) included CTLL-2/IL5R $\alpha$ cells, NK92/NFAT-luciferase cells, and benralizumab (35 ng/mL) 
in $2.5 \%$ NHS to represent the ADCC signal of benralizumab (no ADA inhibition). Low QC (LQC) or high QC (HQC) included CTLL-2/IL5R $\alpha$ cells, NK92/NFAT-luciferase cells, benralizumab (35 ng/mL), and goat anti-benralizumab polyclonal positive control antibody (40 ng/mL for LQC and $100 \mathrm{ng} / \mathrm{mL}$ for HQC) in $2.5 \%$ NHS to represent the neutralization of benralizumab by a polyclonal positive control antibody.

\section{Screening Assay Cut Point Determination}

To determine the assay cut point, a panel of 60 individual serum samples (30 normal serum and 30 asthma serum) of mixed gender along with a pooled NHS sample were analyzed in two separate assays (one on each of 2 days) by two operators. The data set used for the cut point determination consisted of 120 normalized responses (signal to background $[S: B]$ values). To avoid inflation of the cut point, a boxplot outlier test was conducted on the raw data using three inter-quartile ranges (IQRs) as an outlier criterion. Among the 120 data points, four outliers were identified. A normality test was performed on the remaining data points to assess the suitability of using a parametric method to determine the cut point. The test indicated that the data violated the assumption of normality $(p<0.0001)$. The original data $(n=120)$ were then log transformed and no outliers were identified. The 120 data points were then used to test for normality. The normality assumption was again violated $(p<0.0001)$. Subsequently, a nonparametric approach using the log-transformed data was used to determine the cut point (11).

\section{Sensitivity}

The affinity purified surrogate polyclonal and monoclonal anti-benralizumab idiotype antibodies were spiked into the NHS pool to achieve final concentrations of 800, 400, 200, $100,50,25,12.5$, and $6.25 \mathrm{ng} / \mathrm{mL}$. Assay sensitivity was determined from the data of three independent curves for each surrogate ADA control. The lowest concentrations of the polyclonal and monoclonal anti-benralizumab idiotype antibodies in the sample, which were still positive below the cut point, were reported as the estimated assay sensitivity.

\section{Drug Tolerance}

Samples containing $10,5,2.5,1.25,0.63,0.31$, and $0 \mu \mathrm{g} /$ $\mathrm{mL}$ of the polyclonal anti-benralizumab idiotype antibodies were pre-incubated with $12.5,6.25,3.13,1.56,0.78,0.39$, and $0 \mu \mathrm{g} / \mathrm{mL}$ of benralizumab at RT for $60 \pm 5 \mathrm{~min}$ for binding to occur and then frozen at $-80 \pm 10^{\circ} \mathrm{C}$ overnight to mimic sample handling procedures. The samples were mixed with cells and analyzed, and the results presented as $S / B$ ratio. The $S / B$ ratio was calculated based on the RLU of each spiked sample divided by the RLU of the pooled normal serum (NQC).

\section{Selectivity Test}

Ten individual serum samples from asthma patients of mixed gender along with the normal serum pool were tested unspiked (no polyclonal anti-benralizumab idiotype antibodies added) and spiked with $2000 \mathrm{ng} / \mathrm{mL}$ of polyclonal antibenralizumab idiotype antibodies (final concentration is $50 \mathrm{ng} / \mathrm{mL}$ after 40 -fold dilution). The unspiked and spiked samples were each analyzed in one replicate (two wells). The $S / B$ ratio of each individual spike was calculated based on the RLU of each spiked sample/the RLU of the pooled normal serum (NQC).

\section{RESULTS}

The cell-based assay for benralizumab NAb is an ADCC assay, consists of target cells (CTLL-2, a murine cytotoxic T cell line stably transfected with human IL5R $\alpha$ ) and the effector cell (NK92, human natural killer cell line, was engineered to express CD16 (FcRIII $\gamma$ ) and a luciferase reporter gene driven by the nuclear factor of activated $\mathrm{T}$ (NFAT) cells promoter) (Fig. 1). Benralizumab binds to human IL-5R $\alpha$ expressed on the cell surface of CTLL- 2 cells, and the $\mathrm{Fc}$ region of the bound antibody binds to NK92/NFAT cells via CD16 (FcRIII $\gamma$ ), inducing ADCC of the target cell. The ADCC activity is highly correlated with activation of the NFAT transcription factor. The amount of luciferase generated is measured by a sensitive test using the Steady-Glo ${ }^{\circledR}$ luciferase assay system. The luminescence signal is proportional to the ADCC activity and inversely proportional to the levels of NAb present in serum samples.

\section{Target (CTLL-2/IL5R $\alpha$ ) to Effector (NK92/NFAT-Luciferase) Cell Ratio}

To optimize the ADCC activity, different target to effector cell ratios at $1: 1,2: 1,3: 1,4: 1,5: 1$, and $6: 1$ were evaluated. The signal (from the highest concentration of benralizumab) to noise (the lowest concentration of benralizumab) ratio was calculated for each of these cell ratios. As shown in Fig. 2, the luminescence signal increased in a concentration-dependent manner when benralizumab was used from 0.68 to $350 \mathrm{ng} / \mathrm{mL}$ in the assay medium. The target $\left(6 \times 10^{4} /\right.$ well $)$ to effector cell ratio $\left(2 \times 10^{4} /\right.$ well $)$ of $3: 1$ produced a maximal signal to noise ratio, 8.0, which is calculated by the RLU of benralizumab at $350 \mathrm{ng} / \mathrm{mL}$ divided by the RLU of benralizumab at $0.684 \mathrm{ng} / \mathrm{mL}$, demonstrating that 3:1 was the optimal target to effector cell ratio in this ADCC reporter assay.

\section{CTLL2/IL5R $\alpha$ Target Cell Engagement via Binding of Benralizumab Is Essential for Activation of NK92/NFAT-Luciferase Cells}

One of the hallmarks for ADCC activity is target cell engagement via binding of the antibody, which leads to activation of effector cells. To investigate whether binding of benralizumab to the target CTLL2/IL5R $\alpha$ cells is required to activate effector NK92/NFAT-luciferase cells, we incubated benralizumab with NK92/NFAT-luciferase cells alone (in the absence of CTLL2/IL5R $\alpha$ cells). As expected, benralizumab failed to activate NK92/NFAT-luciferase cells in the absence of CTLL2/IL5R $\alpha$ cells (Fig. 3a). Likewise, benralizumab Fab did not activate NK92/NFAT-luciferase cells even in the presence of CTLL2/IL5R $\alpha$ cells. These data demonstrated 


\section{Effector Cell (NK92/NFAT)}

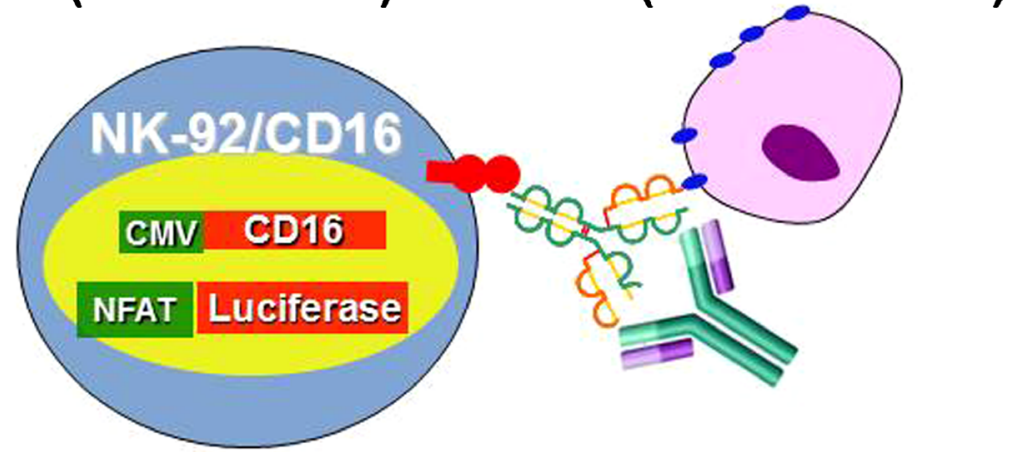

Target Cell (CTLL2/hIL5R)

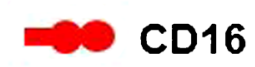

IL5R

Benralizumab

ADA: anti-benralizumab antibody

Fig. 1. Design of the ADCC-based reporter gene NAb assay. The mixture of the diluted serum sample and benralizumab was pre-incubated in a 96-well microplate. CTLL-2/hIL5R cells and NK92/NFAT cells were washed, counted, and then added to each well of the plate. The mixture of the serum sample, benralizumab, and cells was incubated for $4.5 \mathrm{~h}$. Steady-Glo ${ }^{\circledR}$ was added to each well. The plates were then read by a luminometer (PerkinElmer EnVision ${ }^{\mathrm{TM}}$ Multilabel Reader)

that NK92/NFAT-luciferase effector cell activation requires concomitant binding of the antigen binding region of benralizumab to target CTLL2/IL5R $\alpha$ cells.

\section{Benralizumab Demonstrates Enhanced ADCC Activity Through Afucosylation}

Afucosylation of the oligosaccharide core of human IgG1 has been shown to result in a higher affinity to human Fc $\gamma$ RIIIa (CD16) and subsequently an enhanced ADCC $(10,12)$. Benralizumab is a humanized afucosylated antiIL5R $\alpha$ mAb. A previous study has demonstrated that the binding affinity of benralizumab to human FcyRIIIa increased sixfold compared to that of the fucosylated parental benralizumab (13). Moreover, afucosylation heightens ADCC function by more than 1000 -fold over the parental antibody in human peripheral eosinophils (13).

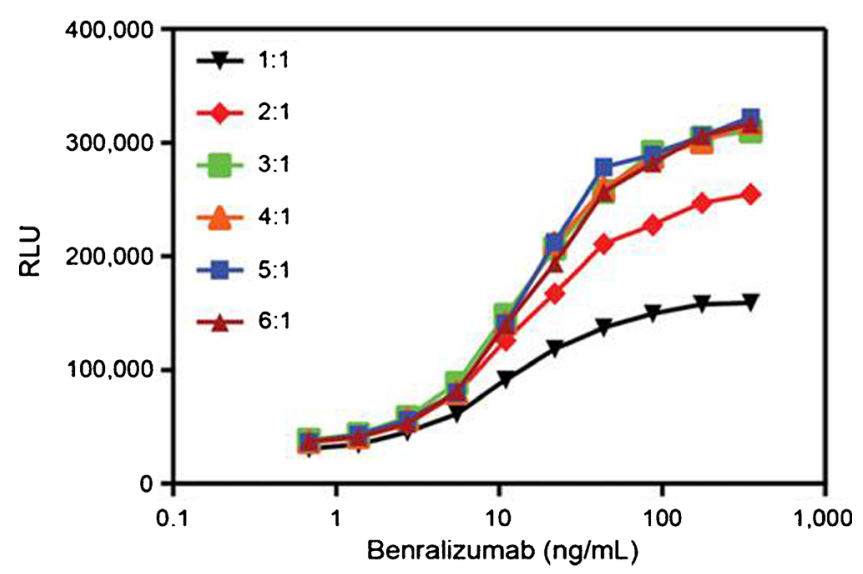

Fig. 2. Target (CTLL-2/IL5R $\alpha$ ) to effector (NK92/NFAT-luciferase) cell ratio. CTLL-2/hIL5R $\alpha$ cells and NK92/NFAT-luciferase cells grown at densities of $2.0 \times 10^{5}$ to $1.0 \times 10^{6}$ cells $/ \mathrm{mL}$ were harvested, prepared at different ratios, and then mixed with benralizumab spiked in assay medium which ranged from 0.684 to $350 \mathrm{ng} / \mathrm{mL}$. The mixture was incubated for $4.5 \mathrm{~h}$, followed by adding Steady-Glo ${ }^{\circledR}$ as a substrate. The results are shown as relative luminescence units (RLU). Each data point was the mean result of duplicate wells. The different symbols represent the different target to effector ratios
We compared the potency of benralizumab and parental benralizumab to mediate ADCC activity in our assay conditions. Benralizumab induced ADCC activity in a dosedependent manner (Fig. 3b), whereas neither parental benralizumab nor IgG1 isotype control was able to do so, indicating that afucosylation significantly enhanced the ADCC activity of benralizumab. Taken together, these data

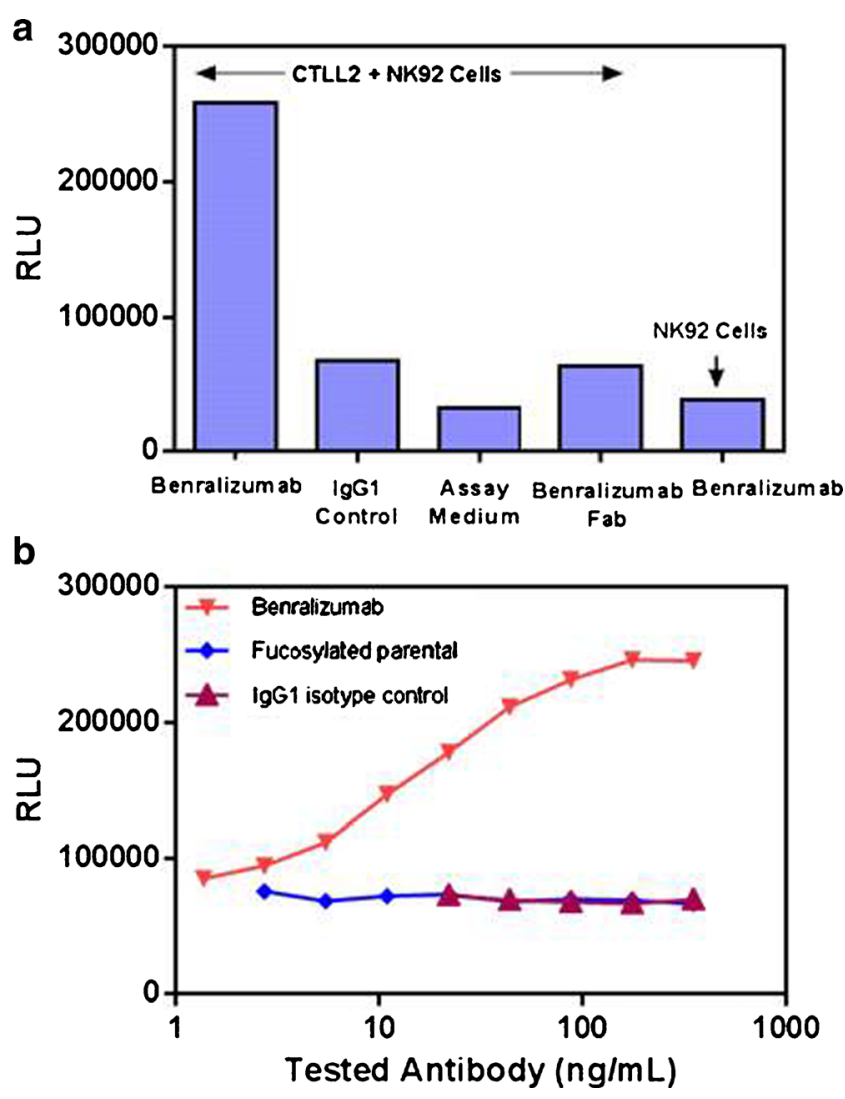

Fig. 3. a, b Characterization of benralizumab-induced ADCC activity. CTLL-2/IL5R $\alpha$ and NK92/NFAT-luciferase were prepared at $6 \times$ $10^{4} /$ well and $2 \times 10^{4} /$ well, respectively. All antibodies were tested at $35 \mathrm{ng} / \mathrm{mL}$. Each data point was generated in duplicate (two wells per sample) 
demonstrated that the assay is only able to function when both the afucosylated Fc (Fig. 3b) and paratope of benralizumab (Fig. 3a) are able to engage the Fc receptor and the IL5R $\alpha$, respectively.

\section{Assessment of Serum Tolerance of CTLL-2/IL5R Cells and NK92/NFAT-Luciferase Cells}

In addition to the drug product in the matrix, other components present in the test serum may also interfere with the ADCC assay; therefore, diluting the serum may help minimize matrix interference. To explore the impact of the matrix on the ADCC assay, benralizumab was spiked into different dilutions of pooled NHS and assay medium $(0 \%$ NHS) at a concentration of $175 \mathrm{ng} / \mathrm{mL}$, and 1:2 serial dilutions were performed. As shown in Fig. 4a, ADCC activity was completely abolished by $10 \%$ serum and partially retained in $5 \%$ serum. The strongest activity was observed in $0.5 \%$
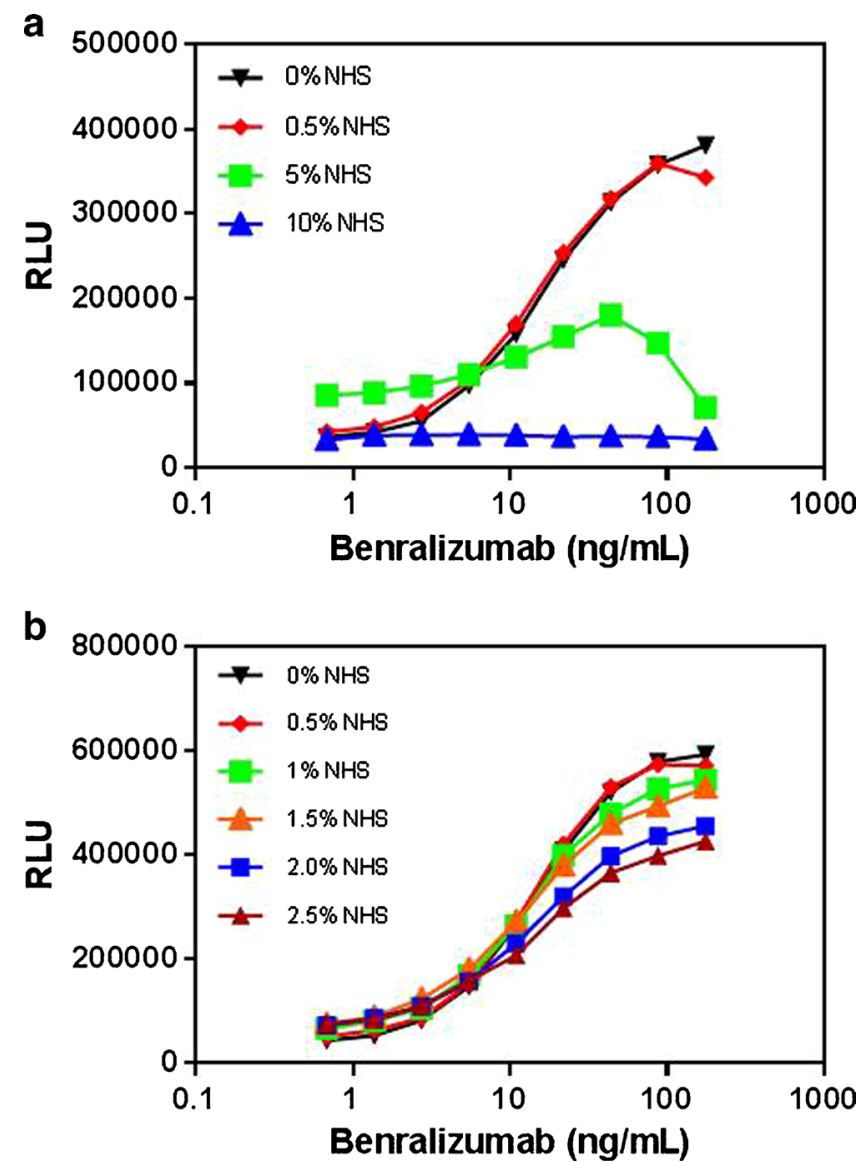

Fig. 4. a, b Evaluation of serum tolerance for CTLL-2/IL5R $\alpha$ and NK92/NFAT-luciferase cells in the ADCC reporter NAb assay. CTLL-2/hIL5R $\alpha$ cells and NK92/NFAT-luciferase cells were harvested and prepared at a ratio of 3:1 (CTLL-2/hIL5 $\alpha$ at $6 \times 10^{4} /$ well and NK92/NFAT-luciferase at $2 \times 10 \%$ well). Benralizumab was spiked into different dilutions of pooled NHS $(0.5 \%, 5 \%$, and $10 \%$ in Fig. $3 a$; $0.5 \%, 1 \%, 1.5 \%, 2 \%$, and $2.5 \%$ in Fig. $3 b$ ) along with the assay medium ( $0 \%$ NHS used as control) at a concentration of $175 \mathrm{ng} / \mathrm{mL}$, and then a 1:2 serial dilution was prepared. Steady-Glo ${ }^{\circledR}$ was added as a substrate after the mixture of cells and spiked benralizumab were incubated for $4.5 \mathrm{~h}$. The results are shown as RLU. Each dilution was measured in duplicate (two wells) serum, indicating that the lower serum concentration (higher sample dilution) permitted the detection of a more potent ADCC response.

A subsequent experiment was conducted to optimize the test sample dilution at $0.5 \%, 1 \%, 1.5 \%, 2 \%$, and $2.5 \%$ (percentage shown in the final assay format). The assay response tended to be more evident with greater serum dilutions (Fig. 4b). For example, $0.5 \%$ serum (equivalent to 200-fold sample dilution) yielded the most potent response among all tested dilutions (Fig. 4b), but this high dilution factor would result in the lowest sensitivity for NAb. Taken together, $2.5 \%$ serum (equivalent to 40 -fold sample dilution) was chosen to balance the assay response, sensitivity, and dynamic window.

\section{Screening Assay Cut Point in Samples from Healthy Donors and Patients with Asthma}

The cut point was determined with 60 individual human serum samples (30 each in the healthy and asthma populations with mixed genders). The $S / B$ ratio of each sample was calculated based on the RLU of 60 individual serum samples to the RLU of the pooled normal serum (NQC), and the cut point was established for each population. The cut point was determined to be 0.78 and 0.82 for the healthy and asthma populations, respectively (Fig. 5) with a false-positive rate of $1 \%$.

\section{Dose Response of Benralizumab in 2.5\% Human Serum}

Testing of increasing concentrations of benralizumab ( 0.684 to $350 \mathrm{ng} / \mathrm{mL})$ in the presence of $2.5 \%$ serum and at the optimal 3:1 target to effector cell ratio resulted in a linear dose-dependent increase in the assay response (Fig. 6). The maximal drug response ratio, 5.6 [calculation was based on the RLU of the sample spiked with $87.5 \mathrm{ng} / \mathrm{mL}$ benralizumab divided by the RLU of the pooled NHS (no benralizumab)] was achieved with approximately $87.5 \mathrm{ng} / \mathrm{mL}$ benralizumab and then plateaued. Benralizumab at a concentration of $35 \mathrm{ng} / \mathrm{mL}$ elicited a 4.7-fold signal induction over the background, which is approximately equivalent to the $80 \%$ maximal effective concentration $\left(\mathrm{EC}_{80}\right)$. This concentration was chosen to be the standard drug concentration for the testing of NAb.

Effect of Anti-benralizumab Antibody on BenralizumabMediated ADCC Activity and Estimation of Assay Sensitivity

To investigate the neutralization of benralizumabinduced ADCC activity by anti-benralizumab antibody, we prepared the assay at the previously determined optimal conditions (2.5\% serum and 3:1 target to effector cell ratio) and tested the impact of affinity purified polyclonal antibenralizumab idiotype antibody to the ADCC-induced signal. The anti-benralizumab idiotype polyclonal antibodies were spiked into a pooled NHS sample at a final concentration of $800 \mathrm{ng} / \mathrm{mL}$, and 1:2 serial dilutions were performed. In the presence of benralizumab at a constant concentration of $35 \mathrm{ng} / \mathrm{mL}$, the $S / B$ ratios decreased with increasing concentrations of the polyclonal anti-benralizumab idiotype 

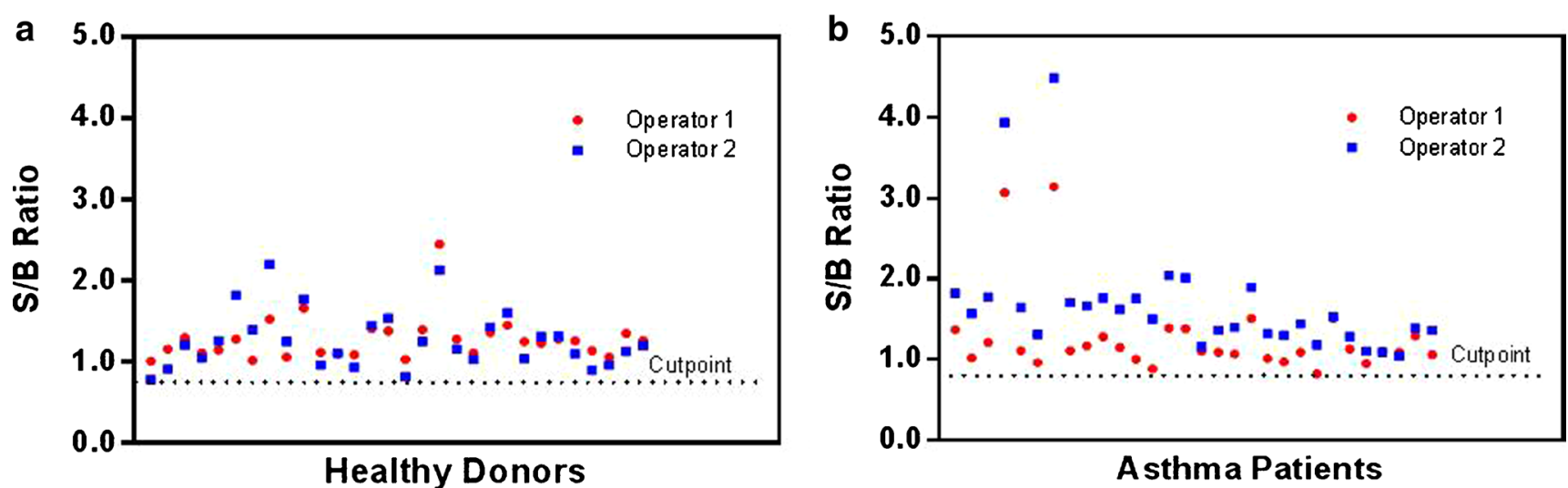

Fig. 5. Determination of the assay cut point from healthy and asthma serum samples. A panel of 30 individual serum samples from each population was analyzed by two analysts according to the assay procedure. Each value represents the $S / B$ ratio [mean RLU sample/mean RLU NHS pool (NQC)] of each well (two wells per sample)

antibody (Fig. 7). The prominent decline in assay response was observed when the polyclonal antibody was at 25 and $50 \mathrm{ng} / \mathrm{mL}$, wherein the molar ratios of the polyclonal ADA to the drug were approximately at $0.7: 1$ and 1.4:1. These results showed that the polyclonal anti-benralizumab idiotype antibody displayed a neutralizing function against the drug, resulting in the inhibition of benralizumab-induced ADCC activity. Furthermore, the polyclonal anti-benralizumab idiotype antibody was shown to be an appropriate positive control for this NAb assay and, thus, could be used to evaluate other assay parameters.

To estimate the assay sensitivity, the polyclonal and monoclonal anti-benralizumab idiotype antibodies were spiked into a pooled NHS sample at a final concentration of $800 \mathrm{ng} / \mathrm{mL}$, and 1:2 serial dilutions were performed. The lowest concentration of the anti-benralizumab antibody in the sample that measured positive (below the cut point) was the

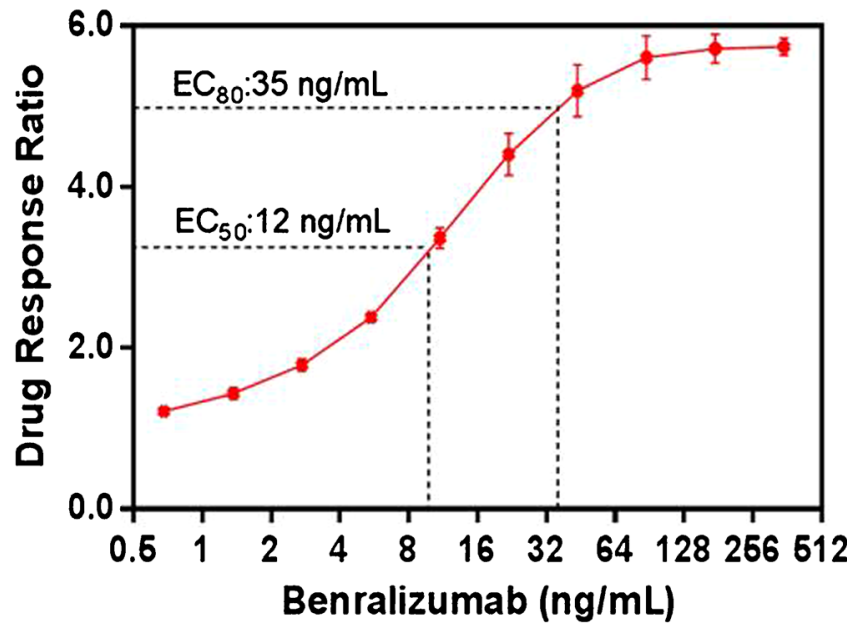

Fig. 6. Drug response curve for benralizumab in $2.5 \%$ human serum. The mean drug response ratios were determined from four assay runs (four runs by one operator on 2 days) and plotted versus the concentration of benralizumab through a four-parameter regression (auto estimate) model. The data are represented as mean $\pm \mathrm{SD}$ values of four determinations (two wells per sample). Drug response ratio: the mean RLU sample spiked with benralizumab/background RLU from the pooled NHS (no benralizumab). The range of background RLU was established and used as one of the criteria to monitor overall assay performance estimated assay sensitivity. As shown in Fig. 7a, the assay sensitivity was estimated to be $1.02 \mu \mathrm{g} / \mathrm{mL}(25.5 \mathrm{ng} / \mathrm{mL}$ polyclonal ADA $\times 40$-fold sample dilution) with the polyclonal anti-benralizumab idiotype antibody. The assay sensitivity was also assessed using a mouse monoclonal antibenralizumab idiotype antibody (known affinity approximately $1 \mathrm{nM}$ ) through a similar approach. The estimated assay

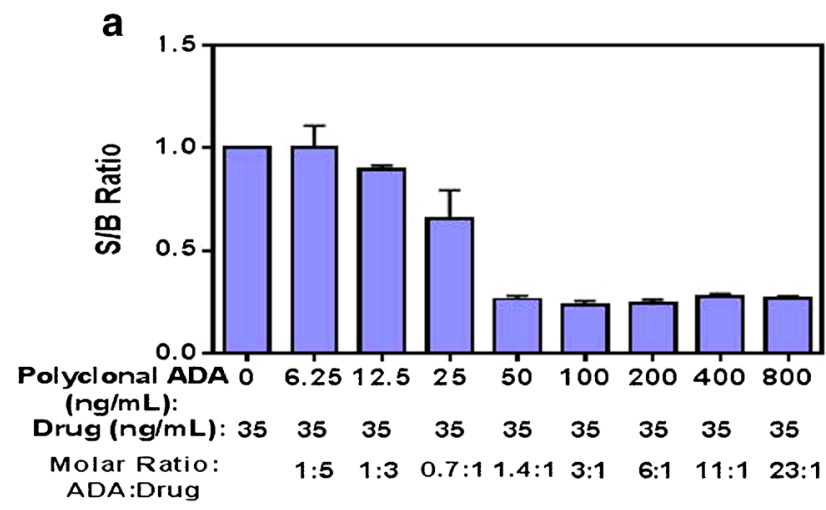

b

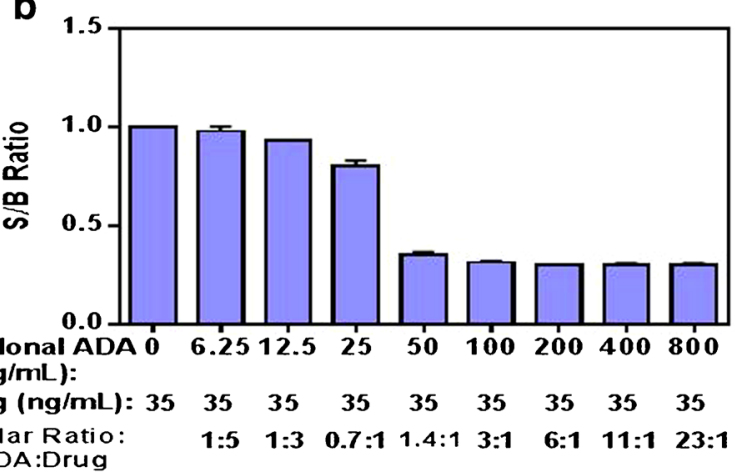

Fig. 7. Inhibition of benralizumab-induced ADCC activity by polyclonal (a) and monoclonal (b) anti-benralizumab antibodies. The anti-benralizumab antibodies were spiked into a pooled NHS sample at a final concentration of $800 \mathrm{ng} / \mathrm{mL}$, and 1:2 serial dilutions were made. Each spiked sample was pre-incubated with a constant concentration of $35 \mathrm{ng} / \mathrm{mL}$ benralizumab at RT for $1 \mathrm{~h}$ before incubating with cells. Data were generated from three runs. $S / B$ ratio: the RLU of each spiked sample/the RLU of the pooled normal serum (NQC) 
sensitivity at the cut point was $1.10 \mu \mathrm{g} / \mathrm{mL}(27.5 \mathrm{ng} / \mathrm{mL}$ monoclonal ADA $\times 40$-fold sample dilution) (Fig. 7b).

\section{Detection of IL5 and SIL5R in Human Serum and Interference of IL5 and SIL5R with the ADCC NAb Assay}

Benralizumab binds to an epitope on $\operatorname{IL} 5 \mathrm{R} \alpha$ that is in close proximity to the IL5 binding site (13). To determine whether IL5 could potentially interfere with benralizumabinduced ADCC activity, we first measured the basal concentrations of IL5 and soluble IL5R (sIL5R) using individual sera from healthy human $(N=30)$ and asthma $(N=30)$ populations (Fig. 8a, b). All the tested samples demonstrated quantifiable concentrations for the endogenous IL5 and sIL5R ranging from 3.63 to $6.80 \mathrm{pg} / \mathrm{mL}$ and 0.640 to $3.92 \mathrm{ng} / \mathrm{mL}$ for the endogenous IL5 (Fig. 8a) and sIL5R (Fig. 8b), respectively. Moreover, there was no significant difference in the concentrations of endogenous IL5 $(p=0.482)$ and sIL5R $(p=0.718)$ between the two populations. We then investigated the potential influence of IL5 and sIL5R on the ADCC assay. Spiked IL5 or sIL5R at concentrations 0.01 to $500 \mathrm{ng} / \mathrm{mL}$ (final concentrations 0.0025 to $125 \mathrm{ng} / \mathrm{mL}$ ) was

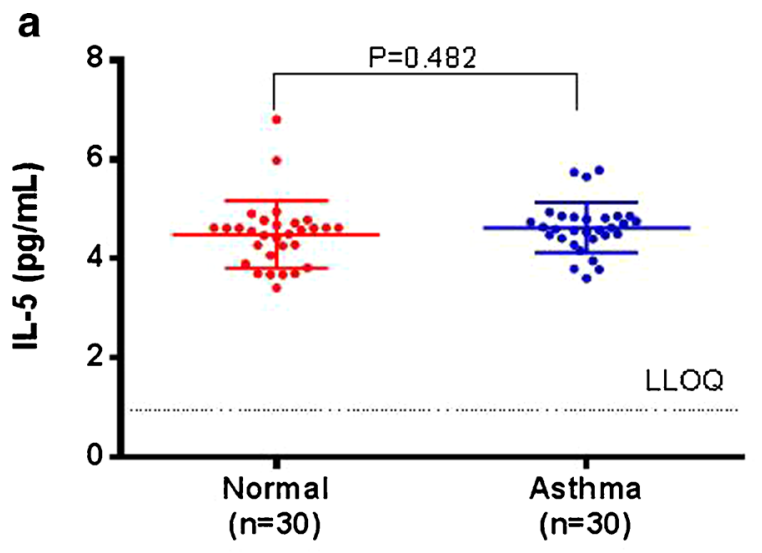

b

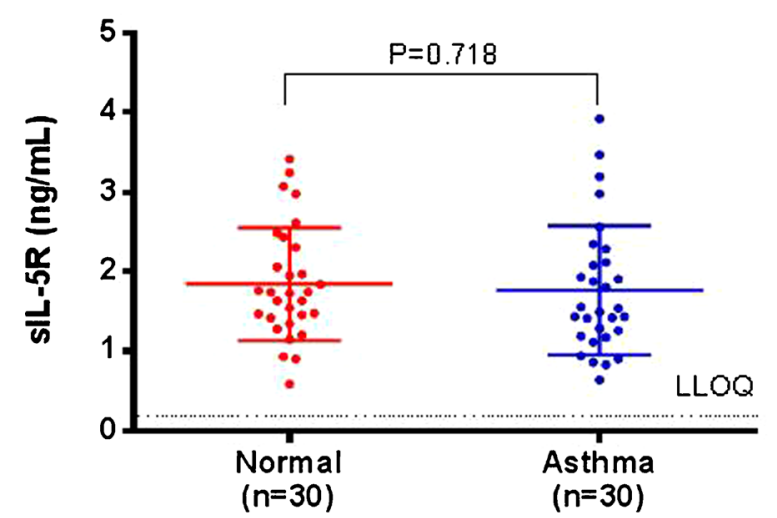

pre-incubated with $140 \mathrm{ng} / \mathrm{mL}$ benralizumab (final concentration $35 \mathrm{ng} / \mathrm{mL}$ ) prior to the assay. As shown in Fig. 8c, the assay signal remained sustainable when IL5 was spiked up to $1.25 \mathrm{ng} / \mathrm{mL}$, then started to drop at $12.5 \mathrm{ng} / \mathrm{mL}$ IL5, and finally came down to background level at $125 \mathrm{ng} / \mathrm{mL}$ IL5. Similarly, the assay signal remained unchanged when sIL5R was spiked up to $12.5 \mathrm{ng} / \mathrm{mL}$, then dropped at $125 \mathrm{ng} / \mathrm{mL}$ sIL5R. These data indicated that neither endogenous IL5 nor sIL5R concentration in normal and disease states affected the benralizumab-induced assay signal given the low concentrations in the serum samples.

\section{Assay Selectivity}

Selectivity of the ADCC reporter cell-based NAb assay was assessed using ten individual serum samples from asthma patients. The polyclonal anti-benralizumab idiotype antibody, at a concentration of $2000 \mathrm{ng} / \mathrm{mL}(50 \mathrm{ng} / \mathrm{mL}$ as final concentration after 40 -fold dilution), was spiked into each individual serum sample along with a NHS pooled sample, and the $S / B$ ratios were calculated. The $S / B$ ratio of each individual spike was below the cut point of 0.82 and was classified positive for benralizumab NAb. These results

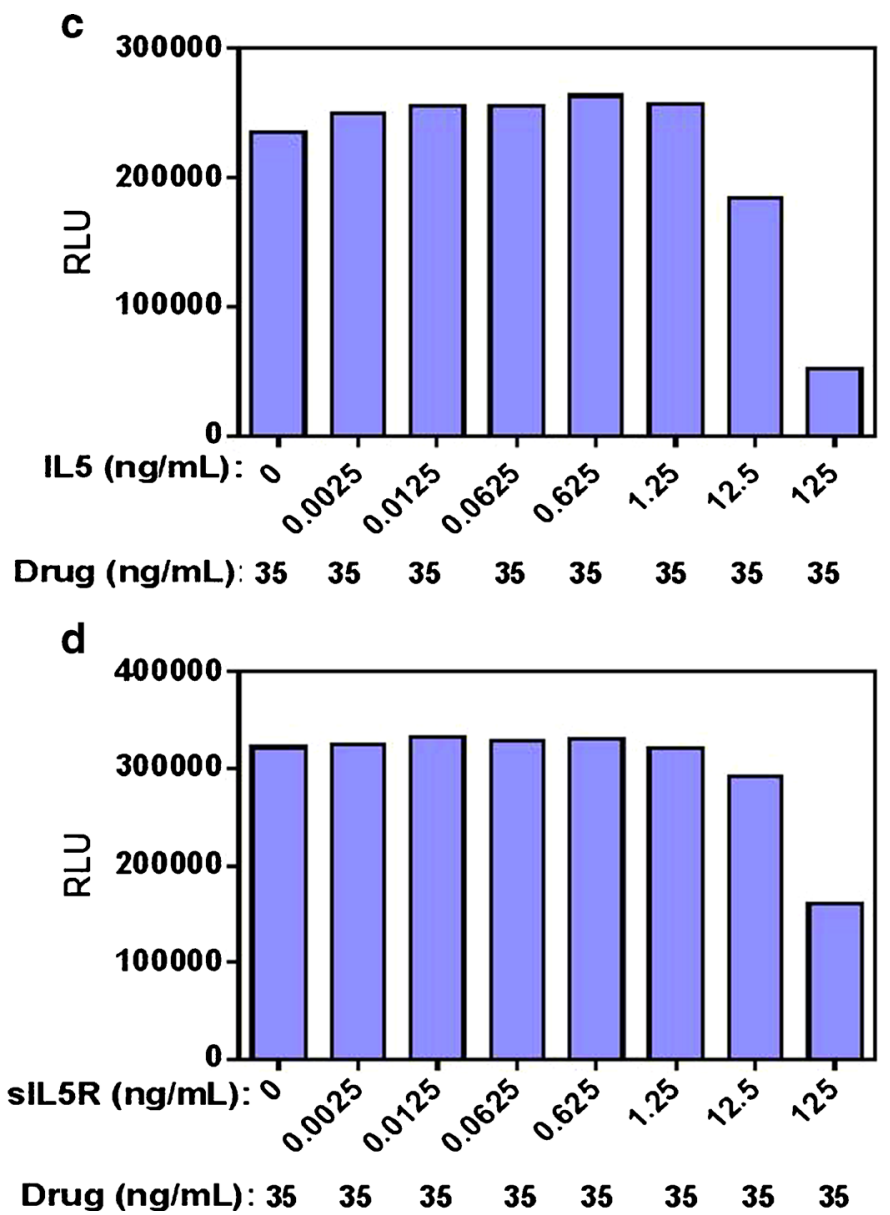

Fig. 8. a and $\mathbf{b}$ IL5 and sIL5R levels in samples from healthy volunteers and patients with asthma. Determinations of IL5 levels from each individual sample were performed according to the procedures described in the packaging inserts for the human IL5 ELISA kit (a). Determinations of sIL5R levels from each individual sample were performed in a sIL5R immunoassay developed by the Bioanalytical Sciences group at MedImmune LLC (b). $\mathbf{c}$ and d. Interference of IL5 and sIL5R with the ADCC NAb assay. IL5 (c) or sIL5R (d) was spiked at various concentrations and then mixed with $35 \mathrm{ng} / \mathrm{mL}$ benralizumab for $30 \mathrm{~min}$ at RT prior to incubating with cells. Each sample was measured in duplicate (two wells) to produce the result 
indicated that the assay was not susceptible to non-specific matrix effects at the final chosen dilution factor.

\section{Drug Tolerance}

Free drug at a fixed concentration is essential for the reproducibility of the $\mathrm{NAb}$ assay as it largely sets the sensitivity for the detection of the NAb. Additional drug in the test sample thus contributes to poor or no drug tolerance by a NAb assay. To evaluate drug tolerance, pooled serum was spiked with the polyclonal anti-benralizumab idiotype antibody ranging from 0.31 to $10 \mu \mathrm{g} / \mathrm{mL}$ and was tested in the presence of benralizumab at various concentrations. The results presented in Table I showed that 2.5 and $5 \mu \mathrm{g} / \mathrm{mL}$ of the polyclonal anti-benralizumab idiotype ADA control were detectable in the presence of $0.78 \mu \mathrm{g} / \mathrm{mL}(0.72 S / B$ ratio) and $3.13 \mu \mathrm{g} / \mathrm{mL}(0.72 S / B$ ratio) of benralizumab, respectively. The data revealed that the polyclonal anti-benralizumab idiotype ADA control to the drug remained approximately at least at 1.6:1 ratio, which may be the threshold for the assay to detect NAb in spiked samples.

\section{Pilot Analysis of Clinical Samples}

To evaluate the ability of the assay to detect NAb in clinical samples, a set of ADA-positive serum samples from three clinical studies were selected and tested in this NAb assay as a pilot study. These 26 samples (selected from 17 patients) were confirmed positive for anti-benralizumab antibodies using a validated electrochemiluminescence ADA confirmatory assay (data not shown), and concentrations of benralizumab ranged from below detection $(<3.86 \mathrm{ng} / \mathrm{mL}, N=$ 11) to $5132 \mathrm{ng} / \mathrm{mL}(N=15)$. Of the 26 samples tested, $8(31 \%)$ samples were shown to be positive for NAbs against benralizumab; 5 out of 8 (63\%) NAb-positive samples had undetectable benralizumab and ADA titers that ranged from 50 to 25,600 (blue, Fig. 9); 3 out of 8 NAb-positive samples had detectable benralizumab with ADA titers that ranged from 50 to 3200 (red, Fig. 9).

Table I. Demonstration of Drug Tolerance in the ADCC Cell-Based NAb Assay

\begin{tabular}{llllllll}
\hline & \multicolumn{7}{l}{ Polyclonal ADA $(\mu \mathrm{g} / \mathrm{mL})$} \\
\cline { 2 - 8 } Drug $(\mu \mathrm{g} / \mathrm{mL})$ & 10 & 5 & 2.5 & 1.25 & 0.63 & 0.31 & 0 \\
\hline 12.5 & 1.14 & 1.22 & 1.22 & 1.24 & 1.38 & 1.30 & 1.25 \\
6.25 & 0.24 & 1.08 & 1.16 & 1.14 & 1.31 & 1.31 & 1.14 \\
3.13 & 0.26 & 0.72 & 1.20 & 1.22 & 1.28 & 1.25 & 1.29 \\
1.56 & 0.25 & 0.27 & 0.91 & 1.14 & 1.16 & 1.08 & 1.24 \\
0.78 & 0.34 & 0.35 & 0.72 & 1.04 & 1.09 & 1.11 & 1.20 \\
0.39 & 0.31 & 0.29 & 0.34 & 0.81 & 1.01 & 0.98 & 1.04 \\
0 & 0.24 & 0.23 & 0.26 & 0.71 & 0.96 & 0.96 & 0.95 \\
\hline
\end{tabular}

Samples containing the polyclonal anti-benralizumab idiotype antibody from 0 to $10 \mu \mathrm{g} / \mathrm{mL}$ were pre-incubated with varying concentrations of benralizumab from 0 to $12.5 \mu \mathrm{g} / \mathrm{mL}$ at RT for $1 \mathrm{~h}$, and then analyzed in the assay. The results are shown as $S / B$ ratios. $S / B$ ratio: the RLU of each spiked sample/the RLU of the pooled normal serum (NQC). The values in italics were below cut point (0.78), indicating a positive result in the assay.

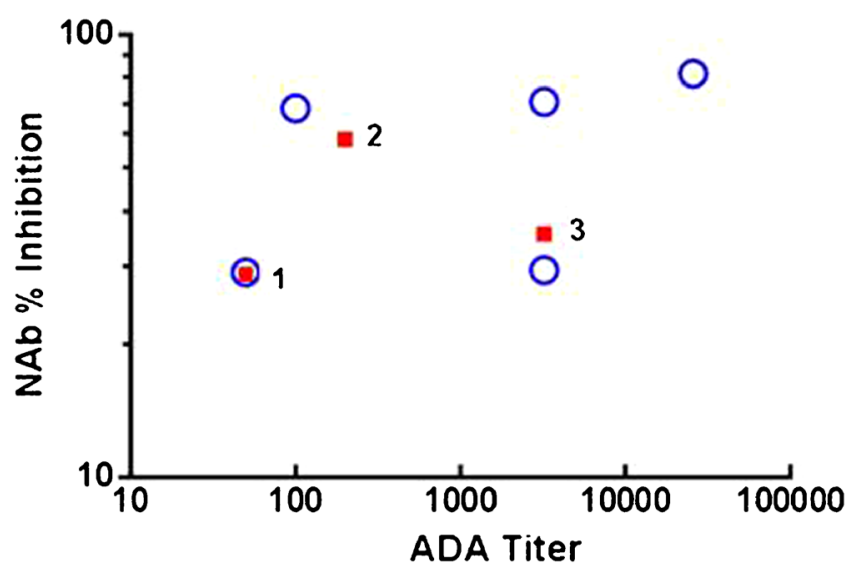

Fig. 9. Anti-benralizumab NAb levels (NAb \% inhibition) and ADA titers in human serum samples from three clinical studies. The 26 serum samples confirmed positive for anti-benralizumab antibodies were subsequently tested for anti-benralizumab NAb. Eight samples were determined to be positive. Blue represents five samples with undetectable benralizumab levels. Red represents three samples with detectable benralizumab levels; sample 1 , sample 2, and sample 3 demonstrate 5132, 395.4, and $6.8 \mathrm{ng} / \mathrm{mL}$ benralizumab, respectively. $\mathrm{NAb}$ percent inhibition was calculated by (1-the mean RLU serum sample/the mean RLU pooled NHS spiked with $35 \mathrm{ng} / \mathrm{mL}$ benralizumab) $\times 100$

\section{DISCUSSION}

NAbs have the ability to bind to the drug product, resulting in abolished drug-associated biological activities as well as cross reaction with the endogenous counterpart (3). As a consequence, characterization of NAbs has become an essential element in evaluating the safety and efficacy of biopharmaceuticals. Regulatory agencies recommend cellbased assays for the detection of NAbs (5); however, the utility of this approach has been limited due to the technical difficulties and practicality of NAb assays $(14,15)$. In parallel to the ligand binding NAb assay, we developed and characterized an ADCC cell-based NAb assay, which is reflective of the MoA of benralizumab to support clinical development of benralizumab.

The ADCC reporter cell-based NAb assay was converted from the potency bioassay, which has been used for potency release and stability testing of benralizumab. During bioassay development, statistical design of experiments (DoE) tools have been implemented to evaluate and optimize several key assay parameters, such as cell densities, assay incubation time, and cell passage numbers, etc. The most optimal condition was established to be a 3:1 ratio for target CTLL2/IL5R $\alpha$ to effector NK92/NFAT-luciferase cells. Incubation duration between 4 and $6 \mathrm{~h}$ was favorable, delivering a reasonably quick turnaround assay (unpublished data). ADCC activities generated from cells with passage at least 40 were comparable to those from cells at initial passage. This was further confirmed during NAb assay development and validation (unpublished data). It is worthwhile noting that the bioassay is performed in formulation buffer, which can fully maximize benralizumab's biological responses.

Given that the NAb assay will ultimately be used to test serum samples, we assessed the cells for serum tolerance. Matrix effects can be a limiting factor in a cell-based NAb 
assay since high concentrations of serum may contain components that might either promote/inhibit cell response or exert cytotoxic effects, leading to false-positive or negative results. Diluting samples is a common and preferred approach to eliminating matrix interference given diluting serum in buffers has minimal impact on bioactivity/viability of cells and sample integrity. The caveat is that assay sensitivity may be compromised due to sample dilution. Most NAb assays employ just one single engineered cell line or tumor cell line (16-18). ADCC is a central MoA for benralizumab; our cellbased NAb assay involves two types of cells, target CTLL2/ IL5R $\alpha$ and effector NK92/NFAT-luciferase cells. This may imply that the ADCC-based NAb assay may require a higher dilution than other $\mathrm{NAb}$ assays where only one cell line is needed (16-18). Despite that CTLL2 cells are more fragile (19) and require high maintenance to achieve good viabilities as compared to NK92 and other engineered or tumor cell lines, our data have shown that 40 -fold sample dilution $(2.5 \%$ serum concentration) provided a favorable condition in which CTLL2 and NK92 cells remained healthy and yielded robust cell response after binding to benralizumab (Fig. 4). The lower serum concentration $(<2.5 \%)$ tended to display a better cell response; however, assay sensitivity would have been comprised if anything lower than $2.5 \%$ serum concentration was chosen.

A series of events is required to initiate ADCC activity. First, the $\mathrm{mAb}$ requires binding to a surface antigen on the target cell. NK cells are then recruited to the target cells via the interaction of FcyRIIIa (CD16) on the NK cell and the Fc portion of the mAb. We characterized the interactions among benralizumab, its target (IL5R on the CTLL2 cells), and effector NK92/NFAT-luciferase cells. Our data clearly demonstrated that target concomitant binding of benralizumab is essential for the activation of ADCC. An earlier study by surface plasmon resonance has demonstrated that afucosylation not only resulted in an increase in the binding affinity of benralizumab for human Fc $\gamma$ RIIIa (sixfold) but also heightened ADCC functions by more than 1000-fold over the parental antibody (13), in which apoptosis as a readout of the ADCC assay was assessed by means of Annexin $\mathrm{V}$ staining in peripheral blood-derived eosinophils and basophils. Our data showed that benralizumab resulted in dose-dependent ADCC activity (Fig. 3). Taken together, these data were in agreement with one another despite the fact that different types of cells and detection methods were employed in the two studies.

IL5 is a competitive antagonist to benralizumab on the IL5R; thus, it is essential to investigate the basal concentrations of IL5 and sIL5R in healthy and asthma serum samples and assess whether they may exert potential effects on the ADCC activity. Although the endogenous IL5 and sIL5R were detected in both tested healthy and asthma serum samples, the quantifiable concentrations ( 3.63 to $6.80 \mathrm{pg} / \mathrm{mL}$ IL5, Fig. 8a; 0.640 to $3.92 \mathrm{ng} / \mathrm{mL}$ sIL5R, Fig. 8b) were far below the concentration which IL5 or sIL5R could interfere with benralizumab-induced ADCC activity (12.5 ng/mL IL5, Fig. 8c; 125 ng/mL sIL5R, Fig. 8d). Neither IL5 nor sIL5R was strikingly elevated in the asthma samples. Moreover, an exploratory analysis for the concentration of sIL5R (unpublished data) using pre- and post-dose clinical samples indicated that sIL5R remained steady as a result of benralizumab administration. These data implied that the likelihood of false response caused by either endogenous IL5 or sIL5R in the blood is very small. Demonstration of no matrix interference with the assay by selectivity data further highlighted the conclusion that the impact of endogenous IL5 and sIL5R on benralizumab-induced ADCC activity is negligible.

It is well recognized that cell-based NAb assays display poor drug tolerance. Regardless of the NAb assay platform, free drug is often used in the assay to induce assay response. This makes it more technically challenging to use NAb assays to detect NAb from clinical samples, even though samples for $\mathrm{NAb}$ testing are usually collected after drug washout. The retrospective pilot study testing clinical trial samples revealed that our ADCC cell-based NAb assay tended to detect NAbs in the high-titer ADA samples but with low or no detectable drug level. Data further supported that our ADCC cell-based $\mathrm{NAb}$ assay has an appropriate sensitivity and reasonable drug tolerance.

Despite the many technical hurdles faced in developing a cell-based NAb assay, we have successfully developed an ADCC reporter cell-based NAb assay that reflects the MoA of benralizumab. The assay performance ensured the success of the validation and has been proven to be adequate and feasible for testing clinical samples. The assay development approach, optimization, and characterization described in this study will shed light on NAb assessment for the class of biopharmaceuticals with ADCC activities.

\section{ACKNOWLEDGMENTS}

This study was funded by AstraZeneca/MedImmune. We would like to acknowledge Jason (Jianchun) Zhang and Harry Yang at MedImmune for providing statistical support.

\section{REFERENCES}

1. Eugen K, Holly WS, Elizabeth S, Gopi S. Recommendations on risk-based strategies for detection and characterization of antibodies against biotechnology products. J Immunol Methods. 2008;333:1-9.

2. Swanson SJ. Immunogenicity issues in drug development. J Immunotoxicol. 2006;3:165-72.

3. Casadevall N, Nataf J, Viron B, Kolta A, Kiladjian JJ, MartinDupont $\mathrm{P}$, et al. Pure red-cell aplasia and antierythropoietin antibodies in patients treated with recombinant erythropoietin. N Engl J Med. 2002;346:469-75.

4. Vultaggio A, Maggi E, Matucci A. Immediate adverse reactions to biologicals: from pathogenic mechanisms to prophylactic management. Curr Opin Allergy Clin Immunol. 2011;11:262-8.

5. Food and Drug administration. DRAFT. Guidance for industry assay development for immunogenicity testing of therapeutic proteins. U.S. Department of Health and Human Services; 2009.

6. Lallemand C, Meritet JF, Blanchard B, Lebon P, Tovey MG. One-step assay for quantification of neutralizing antibodies to biopharmaceuticals. J Immunol Methods. 2010;356:18-28.

7. Ryding J, Hjertberg E, Rasmussen BB. Comparison of two direct neutralizing assay formats using recombinant follicle-stimulating hormone as agonist. J Immunol Methods. 2013;400-1:87-96.

8. Jiang XR, Song A, Bergelson S, Arroll T, Parekh B, May K, et al. Advances in the assessment and control of the effector functions of therapeutic antibodies. Nat Rev Drug Discov. 2011;10:101-11.

9. Chan AC, Carter PJ. Therapeutic antibodies for autoimmunity and inflammation. Nat Rev Immunol. 2010;10:301-16. 
10. Chung S, Quarmby V, Gao X, Ying Y, Lin L, Reed C, et al. Quantitative evaluation of fucose reducing effects in a humanized antibody on $\mathrm{Fc} \gamma$ receptor binding and antibody-dependent cell-mediated cytotoxicity activities. MAbs. 2012;4:326-40.

11. Wu YL, Liu X, Chen Y, Woods R, Lee N, Yang H, et al. An electrochemiluminescence (ECL)-based assay for the specific detection of anti-drug antibodies of the IgE isotype. J Pharm Biomed Anal. 2013;86:73-81.

12. Shinkawa T, Nakamura K, Yamane N, Shoji-Hosaka E, Kanda Y, Sakurada M, et al. The absence of fucose but not the presence of galactose or bisecting $\mathrm{N}$-acetylglucosamine of human IgG1 complex-type oligosaccharides shows the critical role of enhancing antibody-dependent cellular cytotoxicity. J Biol Chem. 2003;278:3466-73.

13. Kolbeck R, Kozhich A, Koike M, Peng L, Andersson CK, Damschroder MM, et al. MEDI-563, a humanized anti-IL-5 receptor alpha $\mathrm{mAb}$ with enhanced antibody-dependent cellmediated cytotoxicity function. J Allergy Clin Immunol. 2010;125:1344-53.

14. Finco D, Baltrukonis D, Clements-Egan A, Delaria K, Gunn GR, Lowe J, et al. Comparison of competitive ligand-binding assay and bioassay formats for the measurement of neutralizing antibodies to protein therapeutics. J Pharm Biomed Anal. 2011;54:351-8.

15. Deehan M, Garcês S, Kramer D, Baker MP, Rat D, Roettger Y, et al. Managing unwanted immunogenicity of biologicals. Autoimmun Rev. 2015;14:569-74.

16. Schwickart M, Mehrzai F, Pearson J, Shaghasi N, Chavez C, Schneider A, et al. Identification and elimination of targetrelated matrix interference in a neutralizing anti-drug antibody assay. J Immunol Methods. 2013;1759:343-8.

17. Sanchez S, Barger T, Zhou L, Hale H, Mytych D, Gupta S, et al. Strategy to confirm the presence of anti-erythropoietin neutralizing antibodies in human serum. J Pharm Biomed Anal. 2011;55:1265-74.

18. Liao K, Sikkema D, Wang C, Chen K, DeWall S, Lee TN. Development and validation of a cell-based SEAP reporter assay for the detection of neutralizing antibodies against an anti-IL-13 therapeutic antibody. J Immunol Methods. 2012;375:258-63.

19. Boise LH, McShan CL, Thompson CB. Introduction of the cell survival gene bcl-xL improves the viability of CTLL-2 cells without affecting their IL-2 proliferative response. Implications for the development of bioassays. J Immunol Methods. 1996;191:143-8. 